\title{
CT arthrography for demonstration of various articular injuries in post-sprained ankle pain
}

\author{
Ashraf Mohamed Hassan El-Sherif, Mohamed Ali Ahmed Mohamed, Nadia Farouk Mohamed El-Ameen, \\ Manal Fayez Abu Samra and Alkawthar Ezedin Saied Abdel-Naby * (B)
}

\begin{abstract}
Background: Post-sprained ankles may sustain ligamentous tear, chondral defect, or osteochondral lesions (OCL). Being widely available and does not depend on high-end machine, the aim of this study was to assess the value of high resolution multi-detector $C T$ arthrography (CTA) in detection of various ligamentous tears, chondral defects, and osteochondral lesions in case of sprain-related persistent ankle pain.

Results: There were 34 (68\%) cases of ligamentous injury, most of which had single ligament affection whereas some cases demonstrated multi-ligamentous injury, and the total number of individual injured ligaments was 42 ligaments. There were 36 cases (72\%) which had either chondral or osteochondral defects; the total number of OCL was 21 lesions and the total number of segmental cartilage defects was 20.

Conclusion: This study emphasized the diagnostic importance of multi-detector CTA in sprain-related ankle pain. In persistent post-sprained ankle pain, multi-detector CTA is a helpful imaging modality which could be utilized for detection of $\mathrm{OCL}$, chondral defects, and various ligamentous tears.
\end{abstract}

Keywords: Ankle sprain, Ligament tear, Chondral defect, Osteochordral lesion, CT arthrography

\section{Background}

Ankle sprain is one of the most common lower extremity injuries; it is responsible for about $10 \%$ of emergency room visits and up to $21 \%$ of sports-related injuries. Lateral sprain is the commonest, accounting for about $85 \%$ of all ankle sprains. Post-sprained ankles may sustain one or more intra-articular injuries as ligamentous tear, chondral defect, and osteochondral lesions (OCL) [1-4].

Non-arthrographic imaging tools including ultrasonography and conventional MRI can be utilized for assessment of ligamentous injuries; however, proper characterization of OCL needs arthrographic-based techniques. CT arthrography (CTA) is a helpful imaging tool which could be utilized in different joints especially the small ones. The advances in CT technologies in last years, principally the multi-channel detector arrays scanners, bring the CTA into the forefront in joint imaging beside the MR arthrography. In the ankle, being a small joint, the imaging technique should have high

\footnotetext{
* Correspondence: alkawthar_ez@outlook.com

Faculty of Medicine, Minya University, Minya 61111, Egypt
}

spatial resolution property, and therefore, CT arthrography might be the study of choice in certain situations in the ankle such as in cartilage imaging and osteochondral lesion assessment, as the ankle has very thin cartilage layer covering its articular surfaces, with their mean ranges from 1.0 to $1.62 \mathrm{~mm}$ in thickness with 0.4 standard deviation [5-10].

Furthermore, CTA can be utilized to detect ligamentous injuries, as it could directly discern ligamentous discontinuity as in anterior talo-fibular ligament (ATFL), or demonstrate extra-articular passage of contrast medium, as into peroneal tendon in case of calcaneo-fibular ligament (CFL) tear [11].

\section{Aim of the work}

Being widely available and does not depend on high-end machine, the aim of this study was to assess the ability of high resolution multi-detector CTA in detection of various ligamentous tears, chondral defects, and osteochondral lesions in case of sprain-related persistent ankle pain. 


\section{Methods}

\section{Study participants}

This study was conducted during June 2017 through June 2018 after being ethically approved by our institution committee. Fifty patients, suffering from sprainrelated ankle pain, were referred from orthopedic clinics, in our hospital, and underwent CT arthrography using Philips 16-detector row machine. Informed written consent was obtained from all patients prior to study.

\section{Inclusion criteria}

Inclusion criteria for patients were based on (1) chronic post-sprain ankle pain lasting more than 3 months either continuously or intermittently. (2) Subacute sprainrelated persistent ankle pain more than 2 weeks duration despite adequate treatment protocol. (3) Recurrent ankle sprain more than three episodes, currently presented with severe acute sprain less than 2 weeks duration.

\section{Exclusion criteria}

Overlying or surrounding soft tissue infection was the only exclusion criterion.

\section{Study techniques}

\section{Ultrasonographic-guided contrast injection}

Ultrasonographic study was the aiding imaging tool, using which the injection of the articular contrast martial was performed. It was done using Xario-200 Toshiba machine with multi-frequency linear array transducer (selected frequency $12 \mathrm{MHz}$ ). The patients were examined while in supine position with the ankle on the examination table. Anterior joint recess evaluation was done for the presence of joint effusion in sagittal and lateral parasagittal planes. Aspiration of any joint effusion was done under careful sterile technique prior to contrast injection.

The injected contrast was non-ionic iodinated contrast medium (Ioversol; Optiray ${ }^{\odot} 350$ ); the contrast medium was diluted 1:1 with lidocaine HCL $1 \%$. The utilized needle was 25 -gauge and $1.5 \mathrm{in}$. length. The ultrasound transducer was placed longitudinally on the anteromedial ankle between the extensor halluces longus and the tibialis anterior tendon, in order to avoid injury of the dorsalis pedis artery; the entry point was $1-2 \mathrm{~cm}$ distal to joint line with $30^{\circ}$ upwards angulation; when the tip of the needle was imaged in the tibio-talar interval, about 8-12 cc of diluted contrast was injected; and the injection was continued until firm resistance to ensure adequate joint distension [12, 13].

\section{CT arthrography}

The patient was scanned in "supine" position using high resolution bone algorithm, thin beam collimation $(0.6 \mathrm{~mm})$, small FOV $(\sim 140 \mathrm{~mm})$, and exposure parameters of $120 \mathrm{kV}$ and $200 \mathrm{mAs}$.

\section{Image processing and analysis}

True axial images were obtained through the long axis of the talus. Coronal reformatted images were obtained along the anterior talar margin. Sagittal images were obtained along the long axis of the calcaneus. Oblique axial images were obtained through the course of the anterior tibio-fibular ligaments $45^{\circ}$ from inferior lateral to superior medial $[12,13]$.

Overview of the entire joint was done using the axial cuts. ATFL, CFL, deep deltoid ligament, and extraarticular passage of contrast were examined on axial plane. Syndesmotic ligaments were assessed on axial oblique images. The coronal and sagittal planes were essential planes for evaluation for chondral defects and OCL $[12,13]$.

OCL classification followed Berndt and Harty staging system modified by Anderson et al. The classification system indicates stage I when the focal compression of the subchondral bone with intact overlying cartilage, stage II when incomplete separation of an OCL, stage IIA means formation of subchondral cyst, stage III when complete detachment of the OCL that remain in situ, and stage IV when complete detachment of the OCL that become displaced.

The ATFL thickness was measured on all cases on true axial images, and it was measured at the thinnest portion in cases demonstrating partial tear, whereas in cases demonstrating intact ligament, it was measured at its mid-portion, cases with complete ATFL tear were not measured as this was not applicable. CFL thickness was measured at its mid-portion in all cases except when torn due to volume averaging from extra-articular contrast within the peroneal tendon sheath, the syndesmotic recess length was measured in coronal plane in all cases from the tibial plafond distally to its top $[12,13]$.

\section{Statistical analysis}

Results of CTA were recorded and tabulated, and the statistical analysis was done using SPSS-16; the data were represented as number and percent. Comparison between ATFL thickness in intact and partially injured cases was done using Mann-Whitney test for non-parametric data. Correlation analysis between pathologic ankle changes and the type of presenting pain was done and Spearman correlation coefficient $(r)$ was assessed. $P$ value $<0.05$ was considered significant.

\section{Results}

There were 23 males and 27 females. The mean age of male patients was $30.83+6.56$ years (range $17-44$ years), 
whereas the mean age of female patients was $37.3+$ 9.18 years (range $22-50$ years).

The most common onset of ankle pain in the study was the chronic onset (29 cases 58\%) and then subacute onset (18 cases $36 \%$ ), the acute onset was present in only three cases, all of which have previous sprain. Recurrent sprain were present in (11 cases $22 \%$ ).

Diffuse ankle pain was the most common presentation (20 cases $40 \%$ ), followed by lateral ankle pain (18 cases $36 \%$ ), and then medial ankle pain (12 cases $24 \%$ ).

The most common type of ankle sprain during presentation was the lateral sprain which was present in 38 cases, whereas 12 cases had medial ankle sprain.

\section{MDCT arthrographic findings}

There were $34(68 \%)$ cases had ligamentous injury on CT arthrography, most of which had single ligament affection whereas some cases demonstrated multiligamentous injury. The total number of individual injured ligaments was 42 ligaments (Table 1).

The ATFL thickness in cases had an intact ligament and in cases which had partial injury are demonstrated in Table 2, and the lower limit of normal was $1.1 \mathrm{~mm}$, whereas in partial injury was $0.3 \mathrm{~mm}$; however, there was a statistically insignificant difference. Mean CFL thickness and syndesmotic recess length are presented in Table 2.

There were 36 cases $(72 \%)$ which had either chondral or osteochondral defects; 18 cases had osteochondral lesions and another 18 cases had chondral injury with intact subchondral bone plate. The total number of OCLs in our study was 22 lesions observed in 18 cases, as there were four cases with talar dome OCL had double OCLs on the medial and lateral corners of the talar dome. The total number of cartilage defects in our study was 20 lesions in 18 cases, with two cases had concurrent tibial and talar cartilage defects (Table 3, Figs. 1, 2, 3, 4, 5, 6, 7, and 8).

Among other arthrographic features, there was one case demonstrating cartilaginous intra-articular bodies, and 21 cases (42\%) had synovial thickening.

Regarding the ultrasonographic features detected before contrast injection, there were 16 cases $(32 \%)$ which had joint effusion, ten of them had synovial thickening.

Among the 34 cases that have ligamentous injury in the study, there were 11 cases complicated with ankle instability; all of them underwent operative treatment and/or ligamentous repair, and all of them were true positive cases proven in operative interventions with no false positive cases. The types of operated ligamentous tears are categorized in Table 4.

Two minor complications happened during arthrographic procedure; four patients developed vaso-vagal attack, as they were sitting rather than lying supine while performing the procedure; and five patients developed mild peri-articular swelling especially anteriorly due to mixed intra- and extra -articular contrast injection. No major complications happened (Table 5).

\section{Correlation analysis}

There was a significant reasonable positive correlation between the presence of OCL or chondral defects and chronic pain presentation, and recurrent sprain (Table 6).

\section{Discussion}

For many years, conventional MRI of the ankle was the traditional imaging study used to assess for ligamentous injuries; however, there are many situations preclude the utility of MRI as in case of presence of a cardiac

Table 1 Different ligamentous injuries in CT arthrography

\begin{tabular}{|c|c|c|c|c|}
\hline \multicolumn{3}{|c|}{ Affected ligament in CT arthrography } & \multirow{2}{*}{$\begin{array}{l}\text { Number } \\
18\end{array}$} & \multirow{2}{*}{$\frac{\text { Percent }}{36}$} \\
\hline$\overline{\text { ATFL }}$ & Partial tear & & & \\
\hline & Complete tear & & 8 & 16 \\
\hline & Total number of ATFL tear & & 26 & 52 \\
\hline & Irregular thickness & $21(80.8 \%)$ & & \\
\hline & Bowing/wavy contour & $5(19.2 \%)$ & & \\
\hline \multirow[t]{6}{*}{ Deltoid ligament } & SD tear & & 2 & 4 \\
\hline & DD tear & & 11 & 22 \\
\hline & Isolated anterior band tear & $3(27 \%)$ & & \\
\hline & Isolated posterior band tear & $4(36.5 \%)$ & & \\
\hline & Both bands tear & $4(36.5 \%)$ & & \\
\hline & Total number of deltoid ligan & & 13 & 26 \\
\hline \multicolumn{3}{|l|}{ CFL tear } & 3 & 6 \\
\hline
\end{tabular}

$\overline{A T F L}$ anterior talo-fibular ligament, $S D$ superficial deltoid ligament, $D D$ deep deltoid ligament, $C F L$ calcaneo-fibular ligament 
Table 2 Some ligamentous morphology on CT arthrography

\begin{tabular}{llll}
\hline Ligament dimension & Intact ligament in $\mathrm{mm}$ & Injured ligament in $\mathrm{mm}$ \\
\hline ATFL thickness & $N=24$ & $\mathrm{~N}=18$ & 0.7 \\
Range & $1.1-2.8$ & $0.3-3.2$ \\
Mean \pm SD & $2.07 \pm 0.54$ & $2.08 \pm 0.88$ \\
CFL thickness & $N=47$ & \\
Range & $1.1-3.7$ & \\
Mean \pm SD & $1.93 \pm 0.46$ & \\
Syndesmotic recess length & $N=50$ & \\
Range & $3.5-23$ & \\
Mean \pm SD & $13.66 \pm 3.9$ & \\
\hline
\end{tabular}

$N$ number, $P$ probability, SD standard deviation, ATFL anterior talo-fibular ligament, $C F L$ calcaneo-fibular ligament

pacemaker, in certain types of aneurysm clips and in cochlear implant; in such cases, CT arthrography would be the excellent solution.

Multi-detector CTA can directly visualize a lot of ankle ligaments, which develop anatomically as intracapsular structures and are normally highlighted by joint fluid. Depending on this fact, most of ankle ligaments could be readily outlined by intra-articular contrast during arthrographic studies, and this highlighting can be easily observed in CTA due to its high spatial resolution. In CTA, the high attenuation of injected contrast medium, in adequately distended joint, could surround the following ligaments: ATFL, PTFL, DDL, and syndesmotic ligaments; therefore, they will appear as hypoattenuated structures, highlighted by high density of injected contrast, and this will not only occur along their inner contours, and also along their outer surfaces. The other ligaments (SDL and CFL) are delineated through their inner surfaces only as they are tightly applied to the joint capsule; their injuries are diagnosed by demonstrating contrast leakage beyond them into adjacent tendon sheaths (i.e., into the tibialis posterior tendon sheath in case of SDL and into the peroneal tendon sheath in case of CFL). Furthermore, CTA is a well-established imaging technique for detection of chondral defects and OCL especially in small joints. CTA of the ankle is considered superior to MR arthrography in hyaline cartilage imaging being very thin in the ankle. MR arthrography has low spatial resolution, and it is unable to separate the closely apposed tibial and talar cartilages and could not detect partial thickness chondral defect. On the other hand, the inherent high resolution of CTA permits easy depiction of the articular cartilage along each articular surface across the joint, and the articular cartilage is clearly seen by virtue of five thin alternating layers of high and low attenuations formed by hyperdense subchondral bone plate, hypodense hyaline cartilage on each side of the joint, and intervening hyperdense contrast material [14-17].

The purpose of the current study was to evaluate the diagnostic value of CTA in post-sprained ankle not only for detection of chondral defects and osteochondral lesions, which are the traditional indications for CTA, but also for detection of concurrent ligamentous tears.

Most of cases in the current study were presented with chronic or subacute ankle sprains with persistent pain,

Table 3 Chondral and OCL lesions and their dimension

\begin{tabular}{lllll}
\hline Pathologic type & & Number of cases & Percent & Maximal dimension in mm \\
\hline Talar OCL & Type I OCL & 2 & 4 & Mean \pm SD \\
& Type II OCL & $11 \pm 2.34)$ & Range \\
& Type III OCL & 11 & 22 & $(7-14)^{*}$ \\
Type IV OCL & 0 & 0 & Mean \pm SD \\
Tibial OCL & Type I OCL & 1 & 2 & $(6.3 \pm 1.93)$ \\
& Type II OCL & 0 & 0 & Range \\
& Type III OCL & 4 & 8 & $(4-8)$ \\
Chondral defect & Type IV OCL & 0 & 0 & 0 \\
& Talar cartilage defect & 7 & 14 & 22 \\
\hline
\end{tabular}

*For type II OCL 


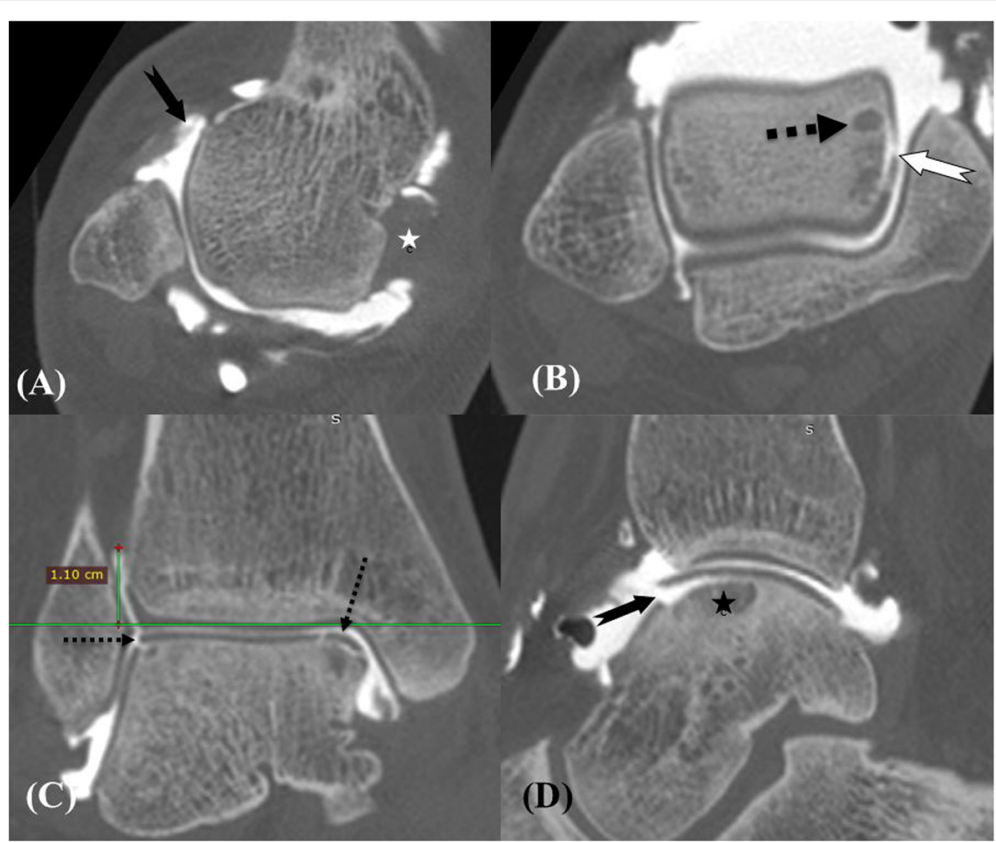

Fig. 1 Right chronic diffuse ankle pain following prior lateral sprain. Axial CTA images through the talus at the level of the talar neck (a) and at the talar dome (b) demonstrate ATFL tear at its talar insertion (black arrow) along with irregular proximal fibers, note normal deep deltoid ligament (star). At the talar dome, there is an extensive OCL with cystic changes (dashed arrow) and delamination of the cartilage (white arrow), Coronal (c) and sagittal (d) CTA show medial and lateral type IIA OCLs (dashed arrows) with significant cartilage delamination (notched arrow) through the medial OCL. Note large osseous component (star). Note syndesmotic recess height measurement on coronal image

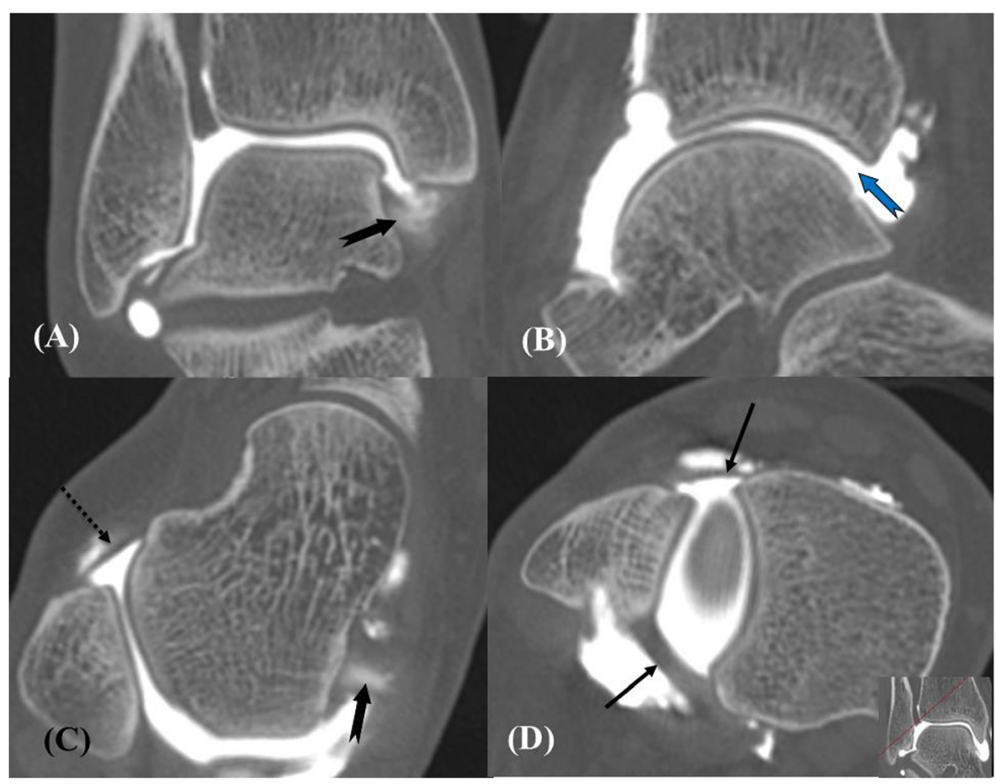

Fig. 2 Right subacute medial ankle pain following medial sprain. Coronal (a) CTA demonstrates contrast medium within the posterior band of the deep deltoid (notched arrow). Sagittal (b) CTA shows uneven joint space that widen posteriorly (blue arrow). Axial (c) CTA demonstrates normal ATFL (dashed arrow). Oblique image though syndesmotic ligaments (d) shows intact anterior inferior tibio-fibular ligament and posterior inferior tibio-fibular ligament 


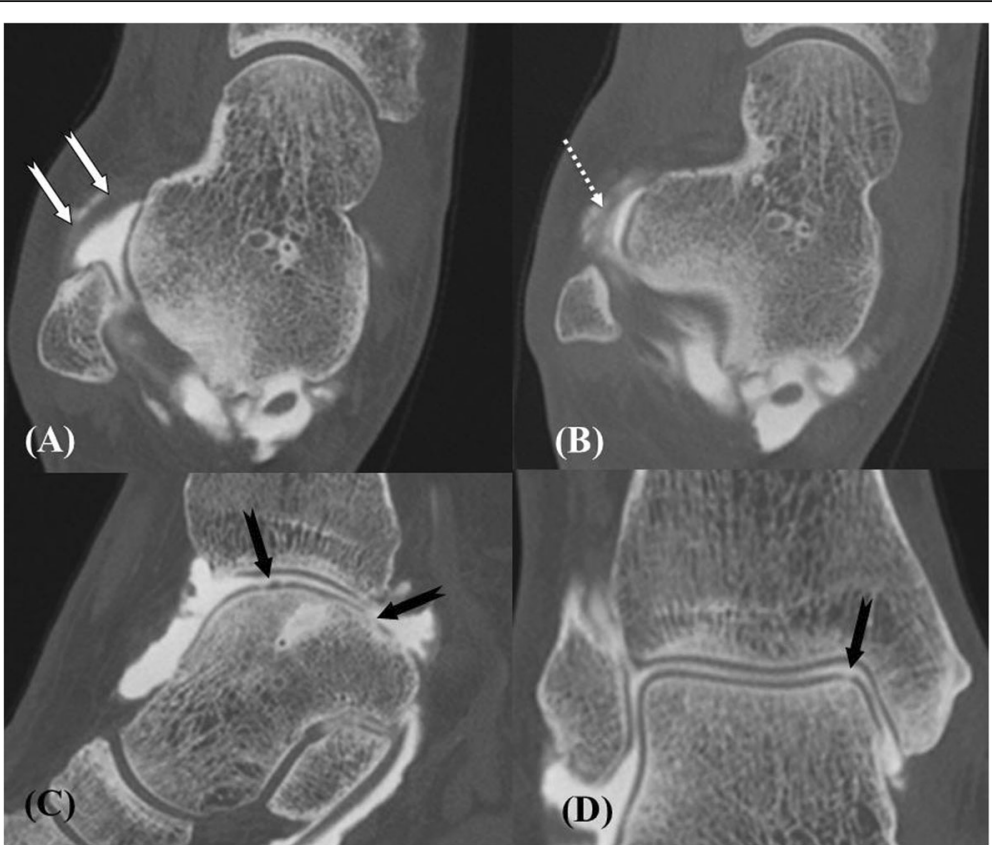

Fig. 3 Right chronic predominately medial ankle pain after multiple episodes of sprains, and the current episode was lateral sprain. Axial CTA images through the talar neck $(\mathbf{a}, \mathbf{b})$ demonstrate lateral bowing of the ATFL, with loss of normal taut appearance (solid arrows), note thinning of the ligament proximally, also note fraying of its distal band (dashed arrow). Sagittal (c) and coronal (d) CTA show cartilage defects through medial and posterior aspects of the talar dome (black arrows) with variable thickness affection, note intact subchondral bone plate and lack of osseous injury. Note also uneven joint space widened anteriorly

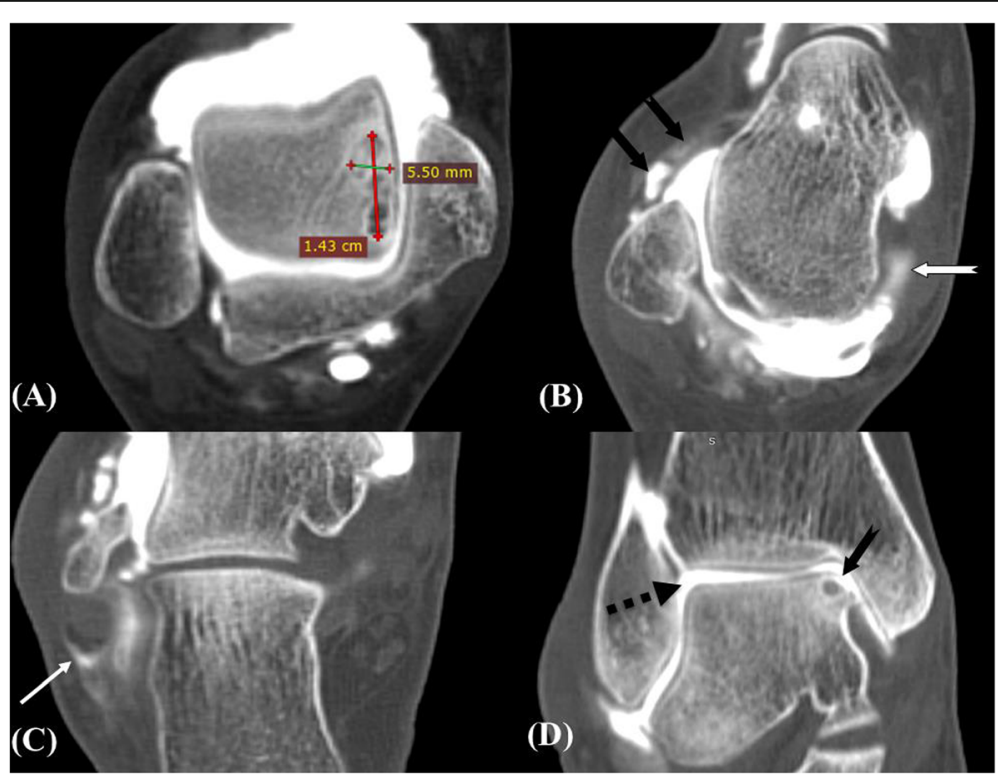

Fig. 4 Chronic diffuse ankle pain after four episodes of ankle sprains, the current episode was lateral sprain. Axial CTA through the talar dome (a) demonstrates large medial OCL with cystic changes (type IIA). Axial CTA through the talar neck (b) demonstrates bowed ATFL (black arrows) with thin fibers at the lateral malleolar attachment. The deep deltoid ligament shows contrast medium within its posterior band (white arrow) indicating its injury. Axial CTA (c) through the calcaneus show contrast within the peroneal tendon sheath indicating CFL tear. Coronal CTA (d) shows uneven joint space that appears widened laterally (dashed arrow), note the cartilage loss overlying type IIA OCL at medial talar corner (notched arrow) 


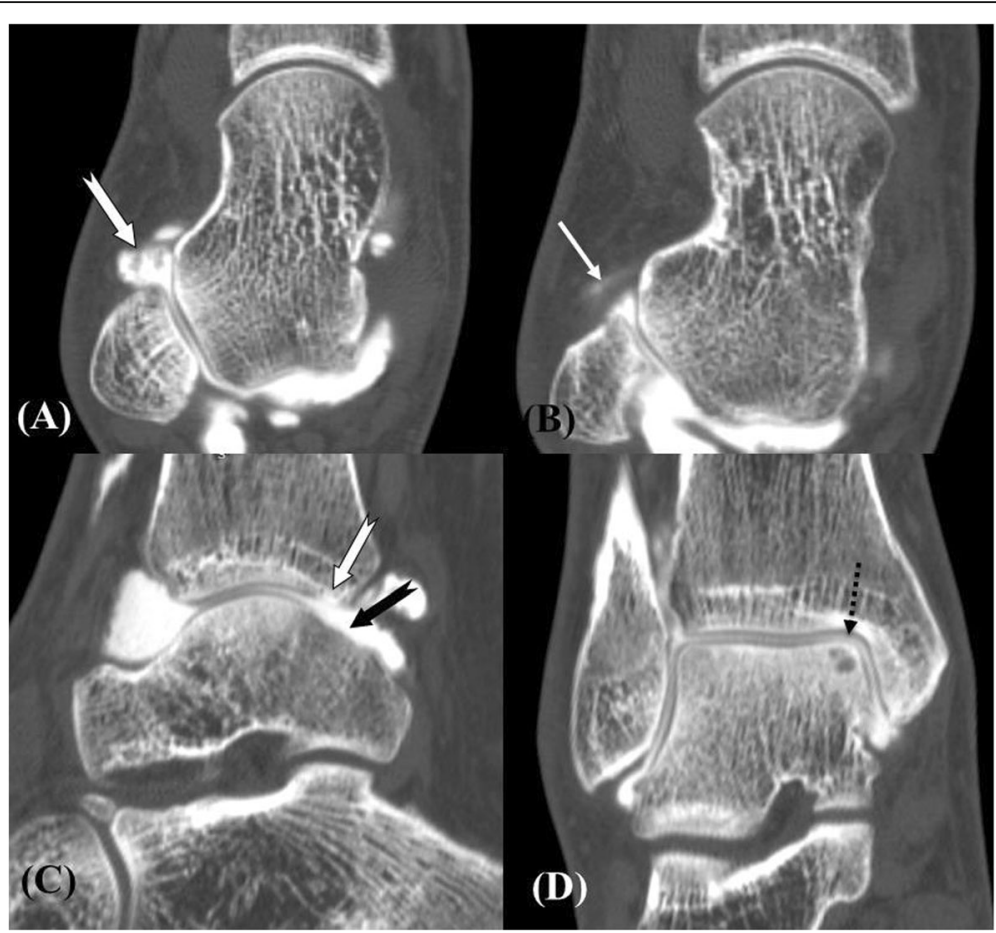

Fig. 5 Acute lateral right ankle pain. He has a history of multiple episodes of lateral ankle sprains; the current episode was lateral sprain. Axial CTA images (a, b) show complete tear of the superior band of ATFL (notched arrow) and intact inferior band (arrow). Sagittal CTA (c) demonstrates wide loss of hyaline cartilage and subjacent bone plate through the posterior talar dome (black arrow) representing type IV OCL, and the subchondral bone appears flattened, note posterior tibial plafond cartilage defect (white arrow). Coronal CTA (d) shows type IIA OCL at the medal talar corner (black arrow)

among the study cases; there were 34 (68\%) which had ligamentous injury detected on CTA, with some had multiple ligament tears. To the best of authors' knowledge, there is lack of studies concerned with the prevalence of ligamentous injuries in sprain-related ankle pain. The high percentage of ligamentous tear found in the current study could be expected particularly in the context of chronic or recurrent sprains, and in such cases, suspicion of ligamentous injury should be raised and kept in mind, as unhealed tear will be a source of

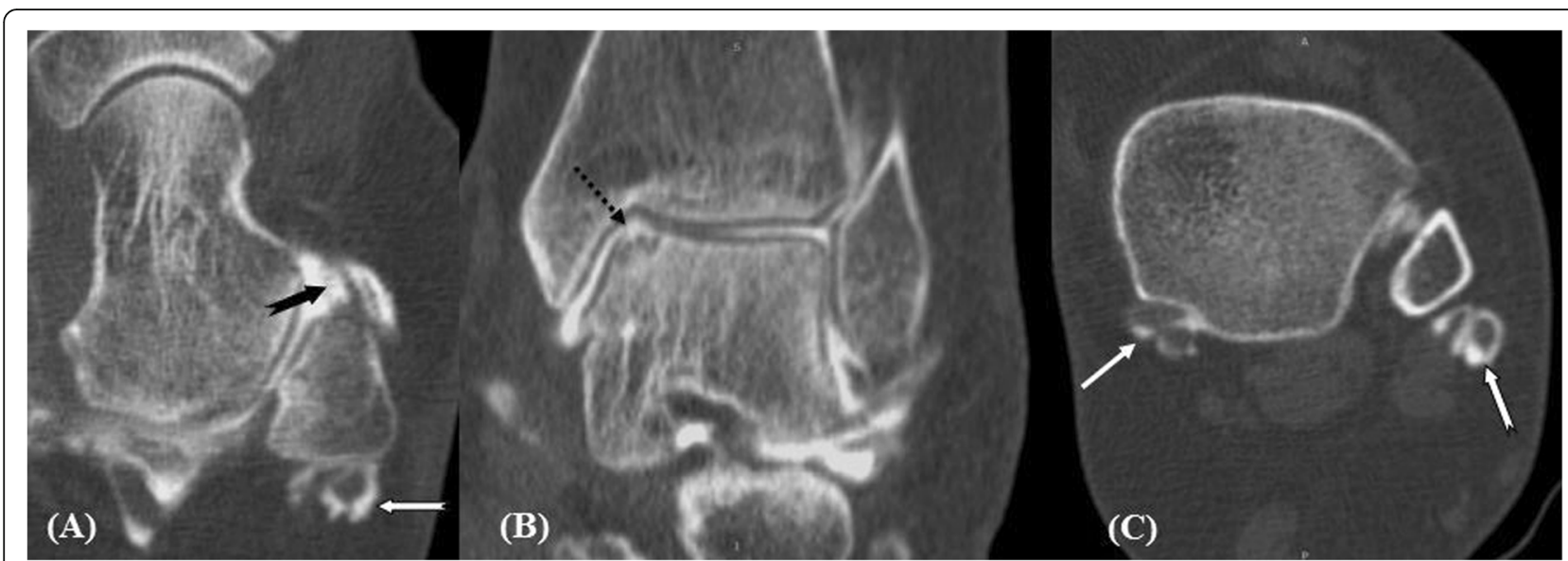

Fig. 6 Subacute recurrent diffuse left ankle pain following severe lateral ankle sprain. Axial CTA image through the talar neck (a) shows a complete tear of the superior band of ATFL (black arrow), and contrast entrance into the peroneal tendon sheath (white arrow) indicative for CFL tear. Coronal CTA (b) demonstrates medial talar dome type IIA OCL (dashed arrow). Axial CTA at the syndesmosis level (c) contrast passage into the tibialis posterior tendon sheath (white arrow) as well as the adjacent flexor digitorum longus tendon sheath denoting superficial deltoid tear through the MMFS 


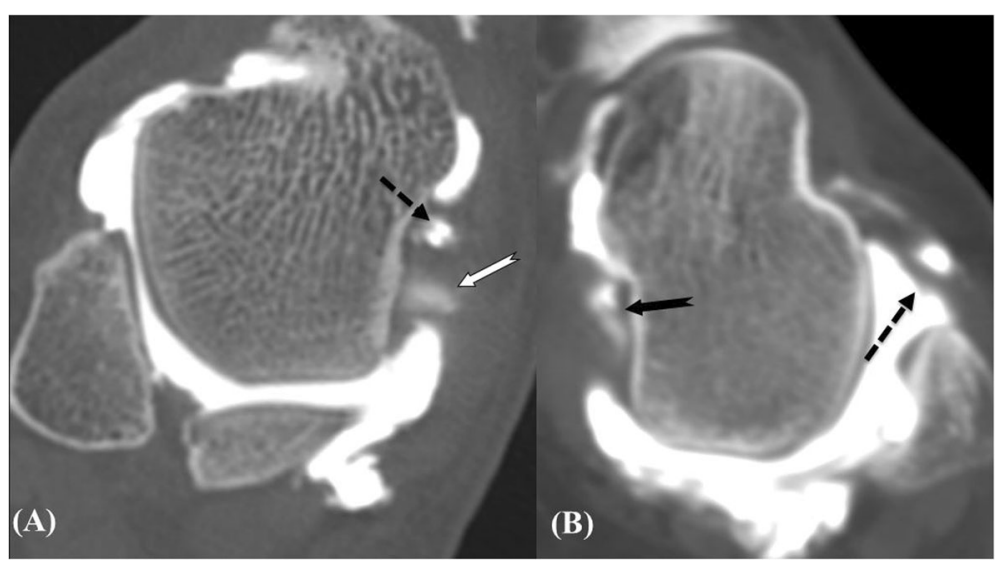

Fig. 7 Axial CTA images of two different patients had previous medial ankle sprain and currently pain. a Both band deep deltoid tear with contrast passage within the anterior (dashed black arrow) and posterior (white arrow) bands. $\mathbf{b}$ Isolated anterior band deep deltoid ligament tear with contrast passage within its fibers (notched arrow), also ATFL irregularity and bowing are present (dashed arrow)

persistent ankle pain and/or leads to abnormal joint motion which in turn leads to ankle instability and development of a vicious circle of recurrent sprains.

Regarding the ligamentous dimensions in the current study, there was no significant difference between partially torn ATFL and intact ones regarding their ligament thickness value (both had mean of 2-mm thickness) although the lower range value was thinner in partially torn ATFL $(0.3 \mathrm{~mm})$ than in intact ligament $(1.1 \mathrm{~mm})$. The mean thickness of normal CFL was $1.9 \mathrm{~mm}$. These data are in concordance with Dimmick et al. 2009, who studied the thickness of ATFL and CFL in normal and abnormal ankles; they utilized MRI in their study and found that the mean thickness of the ATFL was $2.19 \pm 0.6 \mathrm{~mm}$ and the CFL measured $2.13 \pm 0.5 \mathrm{~mm}$, with statistically insignificant difference between normal and abnormal ankles. This statistically insignificant difference between partially torn ATFL and normal ones could signify the utility of the morphologic abnormalities found in torn ligament (such as uneven thickness, bowing, or laxity) as indicators for ligament injury rather than relying on its numerical value of ligament thickness [18].

In this study, there were no cases with high ankle or syndesmotic sprain, all cases demonstrated intact anterior inferior tibio-fibular and posterior inferior tibiofibular ligaments, and the mean syndesmotic recess length was $13.66 \pm 3.9 \mathrm{~mm}$ (range $3.5-23 \mathrm{~mm}$ ). Multiple reports studied the syndesmotic ligaments, however with no thorough documentation or clear cutoff values between normal measurement and abnormal ones. Brown et al. 2004 studied ankle syndesmotic recess by MRI; they found an average recess height of $5 \mathrm{~mm}$ in normal syndesmosis and of $14 \mathrm{~mm}$ and of $12 \mathrm{~mm}$ in chronic and acute syndesmosis injury respectively; these values are seemingly discordant with our results but, this would be attributed to large standard deviation of their means. Moreover, Kim et al. 2007 studied syndesmotic ankle injuries using contrast-enhanced MRI and measured the

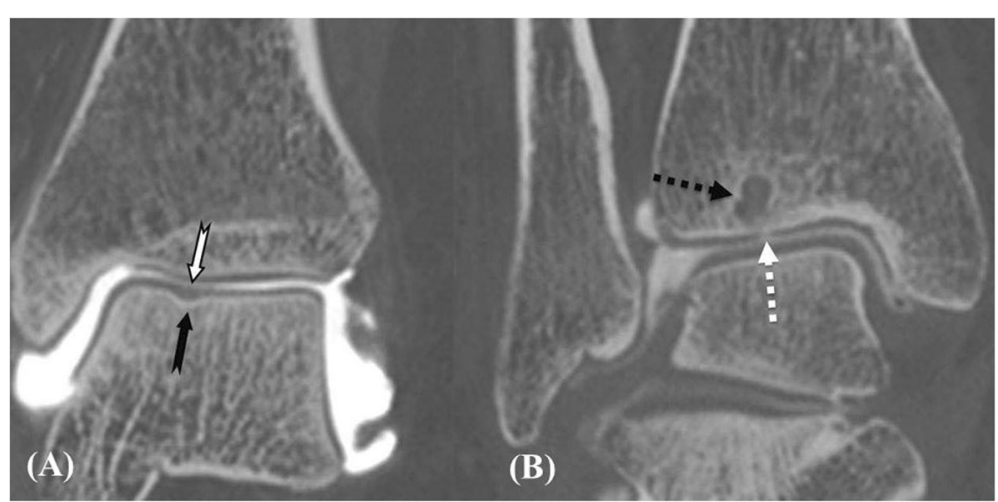

Fig. 8 Coronal CTA images of two different patients had chronic lateral ankle pain. a Focal compression of talar subchondral bone plate (black arrow), with intact overlying cartilage (white arrow) compatible with type I talar OCL. b Focal tibial subchondral cystic changes (black arrow), with overlying cartilage defect (white arrow) compatible with type IIA OCL 
Table 4 Types of surgically proven ligamentous injury

\begin{tabular}{ll}
\hline Ligamentous tear & 8 \\
\hline Complete ATFL tear & 3 \\
Partial ATFL tear & 2 \\
Complete CFL tear & 1 \\
Superficial DL & 1 \\
\hline
\end{tabular}

length of enhancing syndesmotic recess, although they measured the enhanced tissue about the recess, and although they extended the height measurement distally to the talar dome, their results are partially concordant with the current study, they found that the mean height of enhancing tissue delineating the syndesmotic recess was $12.6 \mathrm{~mm} \pm 5.1$ in non-injured ankles whereas it was higher in injured ankle group $(16.2 \mathrm{~mm} \pm 3.3)$ with statistically significant difference. Bartonicek 2003 studied the anatomy of the tibiofibular syndesmosis and found that the expansion of contrast solution as far as $1.2 \mathrm{~cm}$ above the joint could not be considered an indicator of tibiofibular syndesmosis disruption or injury of the interosseous tibiofibular ligament; furthermore, he did not give cutoff value between normal and abnormal syndesmotic height measures [19-21].

Regarding frequency of chondral and OCL in the current study, there were 36 cases $(72 \%)$ which had either chondral defect or OCL; some of which had more than one osteochondral lesions or multi-focal cartilage defect. The occurrence of such lesions in study cases could be expected especially when poor recovery from ankle sprain occurs; however, such high percentage (72\%) of chondral and OCLs might be a little bit surprising, as the indication of CTA in our study was centered about post-sprained ankle pain especially the chronic one which showed a positive correlation with the presence of chondral defect/OCL. The clinical suspicion of chondral/OCL was not an indication for CTA in our study. Kirschke et al. 2016 studied the diagnostic value of CTA for evaluation of osteochondral lesions at the ankle, although the primary indication for CTA in all their cases was to visualize chondral or osteochondral defects; their results are almost in agreement with our study; and they found that $51 / 79(64 \%)$ of patients had talar cartilage defects/OCL and 38/79 (48\%) of patients had tibial cartilage defects/OCL. Furthermore, Kirschke 2016 considered CTA as a reference standard technique

Table 5 Percentage of complications of the arthrographic procedure

\begin{tabular}{lll}
\hline Complication of arthrography & Number (No.) & Percent (\%) \\
\hline Vaso-vagal attack & 4 & 8 \\
Mixed intra- and extra-articular injection & 5 & 10 \\
Infection/septic arthritis & 0 & 0 \\
\hline
\end{tabular}

Table 6 Correlation between presence of OCL or chondral defect and some clinical features

\begin{tabular}{lll}
\hline Clinical feature & $r$ & $P$ \\
\hline Diffuse ankle pain & 0.41 & 0.003 \\
Recurrent sprain & 0.43 & 0.001 \\
\hline$r=0-0.2:$ very low and probably meaningless & \\
$r=0.2-0.4:$ a low correlation that might warrant further investigation & \\
$r=0.4-0.6:$ a reasonable correlation & \\
$r=0.6-0.8:$ a high correlation & \\
$r=0.8-1.0:$ a very high correlation &
\end{tabular}

in order to compare their findings with those of MRI, and they found that MRI was able to visualize only $83.1 \%$ of cartilage defects and $63.6 \%$ of defects of the subchondral bone. In contrast, the current study did not combine CTA with MRI or MR arthrography due to some logistic reasons; it only utilized CTA, in part because of a very thin normal ankle hyaline cartilage and in part due to inherent high resolution of CT images in examining osseous injuries. The high percentage of chondral defect/OCL discovered in the study, together with their positive correlation with chronic ankle pain and with recurrent sprain, could highlight their importance; there should be a high level of expectance of their presence when chronic post-sprained ankle pain workup is initiated $[7,9,22]$.

\section{Study limitation}

Although surgical interventions of some patients in our study allowed for assessment of positive cases, negative case assessment was not available, since operative intervention was primarily targeted to the CTA detected ligamentous tear, and the relatively little number of surgically treated cases is also a limitation. Moreover, although CTA might be considered as a reference for OCL, there was lack of ankle arthroscopy as a reference standard method, as it is not frequently performed in our institution.

\section{Conclusion}

In summary, this study emphasized the diagnostic importance of multi-detector CTA in sprain-related ankle pain. In conclusion, in persistent post-sprained ankle pain, multi-detector CTA is a helpful imaging modality that could be utilized for detection of OCL, chondral defects, and various ligamentous tears.

\section{Abbreviations}

ATFL: Anterior talo-fibular ligament; CFL: Calcaneo-fibular ligament; CTA: CT arthrography; DDL: Deep deltoid ligament; MMFS: Medial malleolar fascial sleeve; OCL: Osteochondral lesions; SDL: Superficial deltoid ligament

Acknowledgements

Not applicable

\section{Authors' contributions}

$\mathrm{AMH}, \mathrm{NF}$, and MF carried out the study design, imaging analysis, and statistical analysis. AE carried out the sonographic assessment of the cases and contrast injection technique. MA carried out all the clinical issues in the study. All authors read and approved the final manuscript. 


\section{Funding}

The study had no funding from any resource.

\section{Availability of data and materials}

The datasets used and analyzed during the study are available from the corresponding author on reasonable request

\section{Ethics approval and consent to participate}

The study was approved by the Research Ethics Committee of the Faculty of Medicine, Minya University, on March 5, 2017; reference number of approval FMREC1764. All cases gave written informed consent to participate in the research.

\section{Consent for publication}

All patients included in this study gave written informed consent for data publishing contained within this study.

\section{Competing interests}

The authors declare that they have no competing interests.

Received: 18 November 2019 Accepted: 2 December 2019

Published online: 20 December 2019

\section{References}

1. Cerezal L, Abascal F, Garcia-Valtuille R, Canga A (2005) Ankle MR arthrography: how, why and when. Radiol Clin N Am 43:693-707

2. Cheung Y, Perrich KD, Gui J, Koval KJ, Goodwin DW (2009) MRI of isolated distal fibular fractures with widened medial clear space on stressed radiographs: which ligaments are interrupted? Am J Roentgenol 192(1):W7. https://doi.org/10.2214/AJR.08.1092

3. Kim M, Choi YS, Jeong MS, Park M, Chun TJ, Kim JS, Young KW (2017) Comprehensive assessment of ankle syndesmosis injury using 3D isotropic turbo spin-echo sequences: diagnostic performance compared with that of conventional and oblique 3-T MRI. AJR 208:827-833

4. Wikstrom EA, Hubbard-Turner T, McKeon PO (2013) Understanding and treating lateral ankle sprains and their consequences: a constraints-based approach. Sports Med 43(6):385-393

5. El-Khoury GY, Alliman KJ, Lundberg HJ, Rudert MJ, Brown TD, Saltzman CL (2004) Cartilage thickness in cadaveric ankles: measurement with double contrast multi-detector row CT arthrography versus MR imaging. Radiology 233:768-773

6. Shepherd DE, Seedhom BB (1999) Thickness of human articular cartilage in joints of the lower limb. Ann Rheum Dis 58:27-34

7. Schmid MR, Pfirrmann CW, Hodler J, Vienne P, Zanetti M (2003) Cartilage lesions in the ankle joint: comparison of MR arthrography and CT arthrography. Skelet Radiol 32:259-265

8. O'Loughlin PF, Heyworth BE, Kennedy JG (2010) Current concepts in the diagnosis and treatment of osteochondral lesions of the ankle. Am J Sports Med 38:392-404

9. Naran KN, Zoga AC (2008) Osteochondral lesions about the ankle. Radiol Clin N Am 46(6):995-1002

10. Kassarjian A (2012) Current concepts in MR and CT arthrography. Semin Musculoskelet Radiol 16(1):1-2

11. Crim J (2018) Lateral ligament injury, ankle. In: Crim J (ed) Specialty imaging Arthrography, vol 8, 2nd edn. Elsevier, Altona, Manitoba, Canada, p 234

12. Crim J (2018) Basic techniques. In: Crim J (ed) Specialty imaging, arthrography, vol 1, 2nd edn. Elsevier, Altona, Manitoba, Canada, pp 2-3

13. Allam MF (2018) Ankle imaging: CT arthrography, In: Allam MF et.al (eds). CT arthrography of the ankle. 1st edn. Scholar's Press, (an imprint of SIA OmniScriptum Publishing), Chisinau, Balti, Riga, Mauritius, p. 21-32

14. Kraniotis P, Maragkos S, Tyllianakis M, Petsas T, Karantanas AH (2012) Ankle post-traumatic osteoarthritis: a CT arthrography study in patients with biand trimalleolar fractures. Skelet Radiol 41(7):803-809

15. Delport AG, Zoga AC (2012) MR and CT arthrography of the elbow. Semin Musculoskelet Radiol 16(1):15-26

16. Allam MF, Elian MM, Allam AF (2018) The value of multi-detector CT arthrography of the wrist joint in evaluation of ligamentous and capsular injuries in post-traumatic pain. EJRNM 49:394-399

17. Crim J, Rosenberg ZS (2016) Ankle. In: Manaster BJ, Crim J (eds) Imaging anatomy. Musculoskeletal, 2nd edn. Elsevier, Canada Altona, Manitoba. Section 11, chapter 51, p 919
18. Dimmick S, Kennedy D, Daunt N (2009) Evaluation of thickness and appearance of anterior talofibular and calcaneofibular ligaments in normal versus abnormal ankles with MRI. J Med Imag Radiation Oncol 52:559-563

19. Brown KW, Morrison WE, Schwetzer ME, Parellada A, Nothnagel H (2004) MRI findings associated with distal tibiofibular syndesmosis injuries. AJR Am Joentgenol 182:131-136

20. Kim S, Huh YM, Song HT, Lee SA, Lee JW, Lee JE, Chung IH, Suh JS (2007) Chronic tibiofibular syndesmosis injury of ankle: evaluation with contrast enhanced fat-suppressed 3D fast spoiled gradient-recalled acquisition in the steady state MR imaging. Radiology 242:225-235

21. Bartonicek J (2003) Anatomy of the tibiofibular syndesmosis and its clinical relevance. Surg Radiol Anat 25:379-386

22. Kirschke JS, Braun S, Baum T, Holwein C, Schaeffeler C, Imhoff AB, Rummeny EJ, Woertler K, Jungmann PM (2016) Diagnostic value of CT arthrography for evaluation of osteochondral lesions at the ankle. Biomed Res Int 3594253. https://doi.org/10.1155/2016/3594253

\section{Publisher's Note}

Springer Nature remains neutral with regard to jurisdictional claims in published maps and institutional affiliations.

\section{Submit your manuscript to a SpringerOpen ${ }^{\circ}$ journal and benefit from:}

- Convenient online submission

- Rigorous peer review

- Open access: articles freely available online

- High visibility within the field

- Retaining the copyright to your article

Submit your next manuscript at $\boldsymbol{\nabla}$ springeropen.com 